\title{
A Novel Biologging Tag that Minimizes the Hydrodynamic Loading on Marine Animals
}

\author{
Aarushi Tiwari \\ Stevenson High School \\ Lincolnshire, Illinois, USA \\ aarushit30@gmail.com
}

\begin{abstract}
Although biologging tags, which are externally attached sensor packages deployed on marine animals, have become essential conservation tools, a core issue with current tag designs is that they are rarely tested for hydrodynamics and may generate substantial hydrodynamic loading (drag and lift forces) on animals. This may cause tags to impede animal physiology, give rise to injuries at the site of attachment, and cause tags to relay unrepresentative data. This study aims to design a new biologging tag form that houses the DTAG3 electronics and reduces the total drag and lift induced on marine animals. One starting model (GPS Phone Tag referred to as Model 0), three iterations, and the final design (Model D), were constructed using CAD software. They were tested with Computational Fluid Dynamics (CFD) simulations to obtain and analyze the drag and lift force. All models were tested at speeds between 1-5 m/s, with 400 trials. The Model D includes a narrow elliptical shape to maintain laminar boundary layers, a pointed tail shape to avoid flow separation, canards for frontal downforce, tabs to reduce form drag, streamlined hydrophones, and dimples to delay flow separation. The CFD simulation results demonstrated that Model D reduced drag by up to $56 \%$ and lift by upto $86 \%$ compared to Model 0 . These results show the potential benefit of this design in reducing the impact of biologging tags on the behavior and
\end{abstract}

energetics of marine animals, and in providing an unbiased and holistic view of the animal behavior for conservation management actions.

Keywords- Animal welfare, biologging, computational fluid dynamics, drag, tag design

\section{INTRODUCTION}

The sophisticated, behavioral data that Biologging tags collect has transformed ocean and aquaculture management [1]. Biologging tags are packages of sensors that can attach to free-ranging animals to relay and study data, giving in-situ insights to features of animal life that are otherwise invisible to ecologists [2]. Furthermore, studying anthropogenic effects on animals has been of particular interest among marine conservationists for assessing the effectiveness of Marine Protected Area borders and fishery equipment; mitigating boat-cetacean collisions and sonar disruption; (is this grammar correct) creating population models for overexploited and endangered fish; and determining fish migratory and feeding grounds. This data about where animals are most vulnerable can be utilized for use in marine management policies [1].

Despite their usefulness, tags act as a disturbance to the flow around streamlined marine animals, which can heighten the energy required to swim, leading to decreased swimming speeds [3]. This severely impacts their reproduction, 


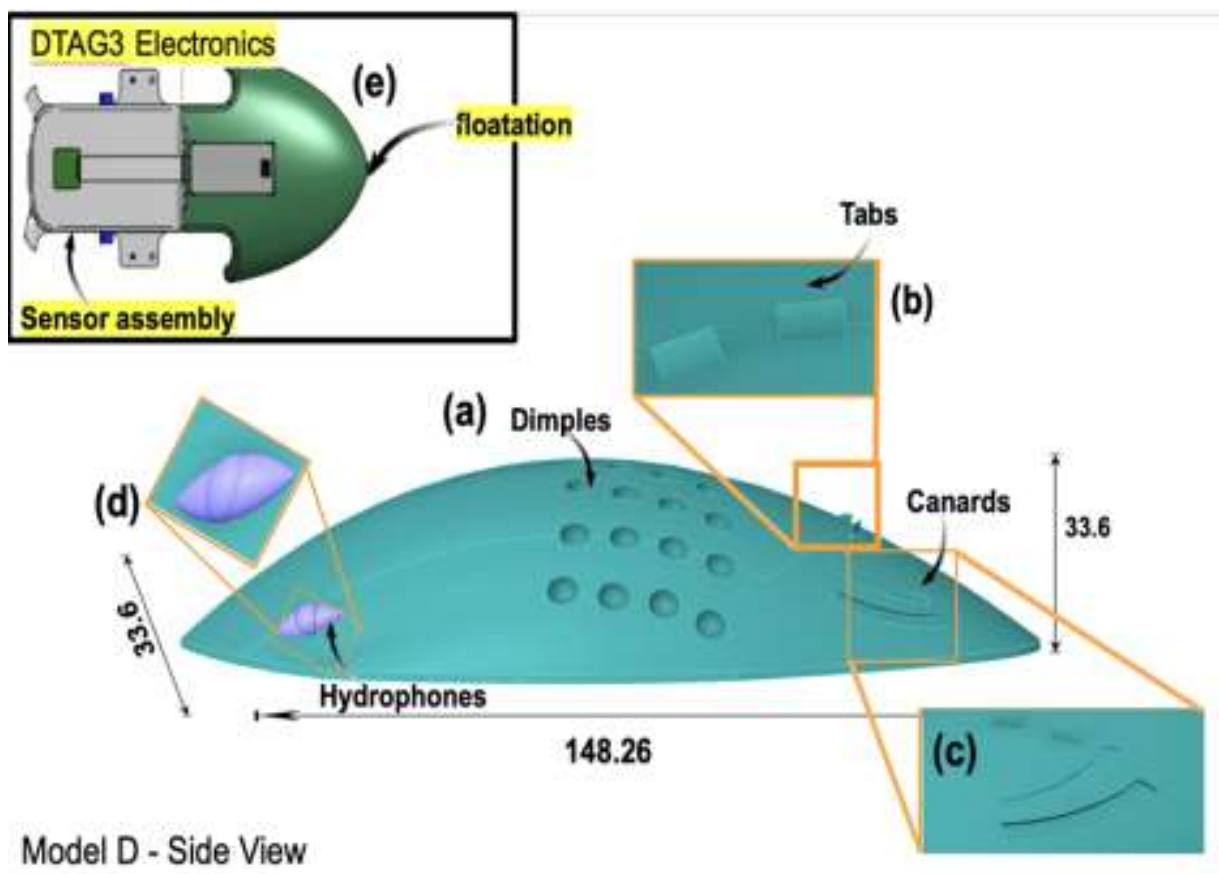

Fig. 1: Model D CAD design (final tag design) in mm, with close up views of the cut-out canards (c), tabs (b), streamlined hydrophones (d) and electronics package (e)

Migration and survival abilities [4, 5, 6, 7] This also means that the data collected by tag can be biased, impacting ocean management decisions $[8]$.

The Biologging tag designs today are not tested for hydrodynamic loadings and its effects on animals. The existing designs generally consider guidelines based on the weight of the tag, such as the $2 \%$ body mass rule, but this method has shown to be a highly simplified rule that neglects the complex hydrodynamics involved [9]. Current tag designs limit themselves to creating the smallest possible encasing around the electronics to meet weight guidelines [10]. However, in-situ studies, recent refinement reports from U.S Fisheries \& Wildlife, and guidelines from the U.K Working Group indicated that tags that have more mass can generate lower amounts of drag and lift $(\mathrm{N})$ ('loading'), if designed with hydrodynamic considerations such as the drag and lift force [11].

The main source of drag is through the formation of a stationary region of water on the surface of the body called a boundary layer [12]. The shape of the tag greatly affects the state of the boundary layer, and kind of boundary layer that is optimal for maximum drag reduction is determined based on what kind of drag is dominant. When the flow is steady, or laminar, the low momentum boundary layer created is prone to moving past the body and separating from the contour of the body, which leads to pressure drag and a low-pressure wake behind the body. When the flow becomes chaotic, or turbulent, high energy vortices are formed, leading to friction drag [12].

Bernoulli's equation gives the underlying mechanism behind lift:

$P+1 / 2 \rho V^{2}=$ Constant

Bernoulli's equation states that the sum of the local static pressure $(P)$ and the dynamic pressure ( $\rho$ is the fluid density and $V$ is the speed of the fluid) is constant along a streamline.

Bernoulli's equation shows that an increase in velocity must be accompanied with a decrease in pressure, and a decrease in velocity causes an increase in pressure along a streamline [12]. Blunt bodies (ex. conventional tag shapes) immersed in moving fluids results in the fluid 
moving faster over the surface, which will lead to a consequent pressure imbalance between the upper and lower surface of the tag [3]. These pressure differentials are the main source of lift [12]. It is important to minimize lift to decrease the tag footprint imparted on the animal, such as abrasions and adverse thermoregulatory responses at the site of attachment [10].

Recently, researchers at Swansea university tested how the shape, size, and position of the tag affects the drag and lift experienced by a tagged grey seal [10]. Furthermore, studies carried out by [3] extended the work of [13], and they investigated the contribution of different "flow control elements" (venturi channels, inverted wing, etc.) to tag induced drag and lift. However, certain elements brought up practicality concerns when translating to real-life settings. Additionally, while their final design elements significantly reduced the lift force, they also accounted for larger drag.

In this study, a new hydrodynamic tag design and three other iterations are tested using Computational Fluid Dynamics (CFD). The new design incorporates practical load reduction elements inspired from the automotive, sports, and submarine industry, and tests reveal that it could significantly reduce both drag and lift induced by the tag. It is believed that this design will help in collecting unbiased data to provide a holistic view of the animal's life, and help in furthering the efforts of protecting oceans and marine life.

\section{METHODS}

\section{TAG DESIGN}

The new tag will house the high-tech DTAG3 electronics package (flotation and senor package). This adds the extra requirement that the drag and lift reduction methods incorporated have to be passive methods, meaning that there would be no actively moving parts on the tag that are dynamically changing its form or any elements of it. Such methods have the possibility of increasing the chances of electronic failure [13]. The new tag is assumed to be attached through glue on the animal, and (Figure 1e) in a watertight casing.
(Model 0) A CAD model of a tag similar in shape and function to the existing Splash309a and Time Depth Recorder (TDR) (see [4] for more details) was made to act as a point of reference to design the new tags. This model is a rectangular prism shape, with a wide, square profile, and it has a hemisphere and half cylinder stationed at the top (Figure 6a).

(Model A) Submarine designers face similar problems to the ones tag manufacturers are facing when trying to make the overall body shape more hydrodynamic, given the electronic constraints [14]. Therefore, to reduce drag, a slowly converging elliptical nose shape was added, inspired from submarine hull design. Such a nose shape generates a laminar boundary layer to avoid friction drag. Since laminar boundary layers are more prone to flow separation, a pointed tail shape would enable the flow to move towards the tail with minimal separation.

(Model B) To induce a downforce that would counteract the lift force by generating high pressure above the tag and redirecting flow up and away from the tag, extruded canards were added at the front of the tag. These are planes commonly seen in race car design [15]. Because canards have a relatively small surface area, they would induce less drag than observed in other downforce generators like spoilers [13].

(Model C) Dimples, similar to dimples on a golf ball, were incorporated (Figure 1a). Through generating a turbulent boundary layer, the vortices created by dimples transfer more momentum to the laminar boundary layer, delaying flow separation and therefore reducing drag. They were added on the tag nearest to the maximum cross-sectional area as that is where the boundary layer has the highest possibility of separating [16].

(Model D) Tabs, which are perpendicular plates, were added behind the canards (Figure 1b). Tabs increase high pressure flow near the front of the body, which decreases both lift and pressure drag in both laminar and turbulent dominant flow settings [17]. The extruded canards were replaced with indented canards (Figure 1c) to avoid any possible entanglements with vegetation. Finally, more streamlined hydrophones were incorporated to further reduce drag (Figure 1d) 


\section{COMPUTATIONAL FLUID DYNA-MICS SIMULATIONS}

CFD has been recognized to be an efficient way to prototype new tag designs and investigate the underlying physical mechanisms behind the new elements, as opposed to conducting tests with wind tunnels or live animals [18]. Indeed, CFD results have been found to be in close agreement with wind tunnel results [3]. One starting model (Model 0) and four iterations, (Model A, Model B, Model C, Model D) were constructed through Computer Aided Design (CAD), and they were tested with CFD simulations, where the software used was Ansys academic ${ }^{\circledR}$ Spaceclaim ${ }^{\circledR}$ and Ansys academic®Fluent version R20 respectively (ANSYS, Inc., Pennsylvania, USA). The simulations were run with a k- $\varepsilon$ turbulence model. Furthermore, the drag and lift forces simulated are defined as:

$C{ }_{D}=\frac{D}{1 / 2 \rho V \quad \stackrel{\infty}{2} A}$

$$
C \quad L=\frac{L}{1 / 2 \rho V \quad 2 \quad A}
$$

Aerodynamic force equations, where $D$ and $L$ is the absolute drag and lift forces $(N)$ of the tag respectively; $A$ is the reference area (surface area); $\mathrm{Cd}$ and $\mathrm{Cl}$ are the drag and lift coefficient respectively; $\rho$ is the fluid density $\left(1028 \mathrm{~kg} / \mathrm{m}^{\wedge} 3\right)$; and $V$ is the freestream velocity $(1 \mathrm{~m} / \mathrm{s}, 3 \mathrm{~m} / \mathrm{s}, 5$ $\mathrm{m} / \mathrm{s}$ ).

The tag was positioned in the middle of a 360 mm (width) x 1500 (length) x 580 (height) enclosure with a defined inlet and outlet flow. There was a surface mesh generated around the whole domain and a refined mesh region around the tag itself. All models were tested at three different speeds of $1 \mathrm{~m} / \mathrm{s}, 3 \mathrm{~m} / \mathrm{s}$, and $5 \mathrm{~m} / \mathrm{s}$, with 400 iterations each (see CFD workflow). These are common speeds marine animals take while descending, ascending and foraging in high tidal environments respectively [18].

\section{CFD Workflow}

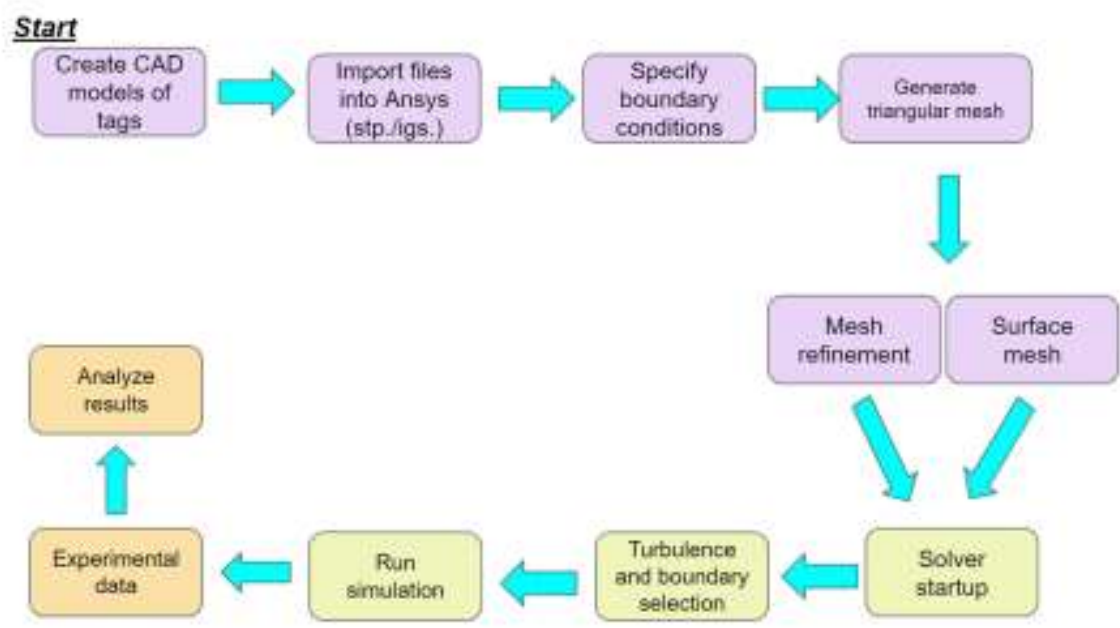

Sinnce the acedemic version of the CFD software limits the user in how many cells the mesh on the top of the body being tested can have, a mesh convergence study wasn't conducted. That is, instead of determining the proper mesh size to receive accurate results (i.e. a mesh convergence study), the finest mesh available had to be used. Refined mesh regions will account for more precise results, but correspondingly larger computation power [18].

I. Simulation Results

Relative comparisons were made between iterations and Model 0 at the highest speed tested $(5 \mathrm{~m} / \mathrm{s})$ as that is when the effects of drag (Figure 2) and lift (Figure 3) are most pronounced, based 


\section{Model 0 Model A Model B Model C Model D}

\section{Drag (N) vs. Tag Design}

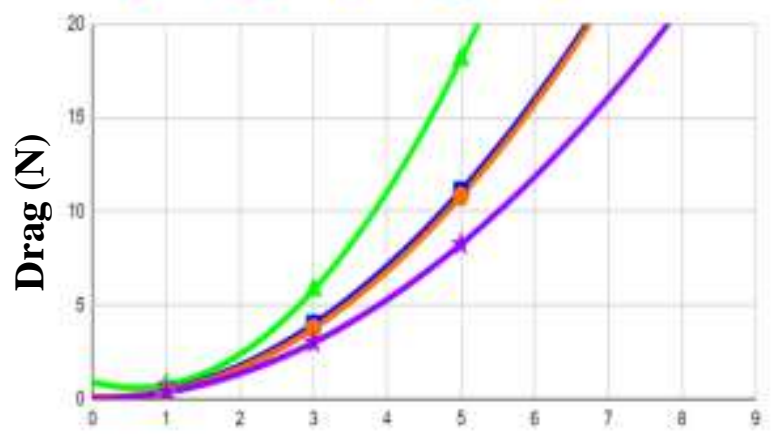

Velocity $(\mathbf{m} / \mathbf{s})$

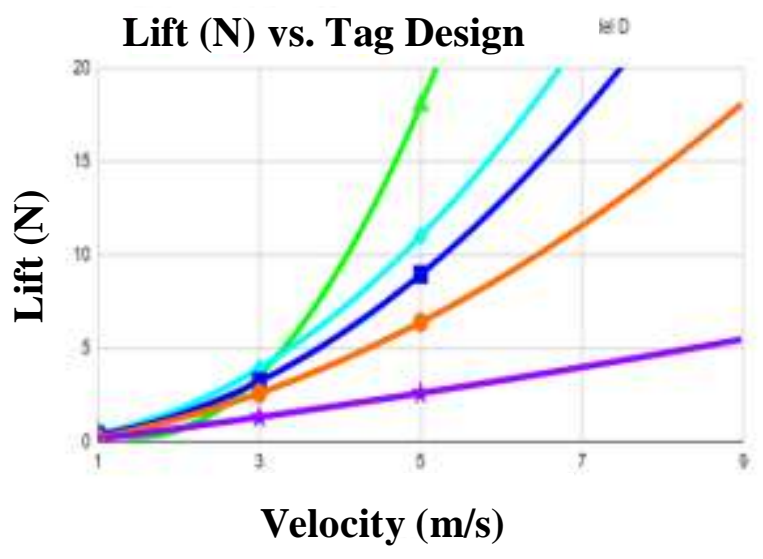

on the force values computed by the simulations (See Appendix A for data table).

Model 0 (control variable) generated a drag force of $\approx 18.1 \mathrm{~N}$ a lift force of $\approx 18 \mathrm{~N}$. Furthermore, a large region of stagnation pressure at the front of the tag formed, creating an adverse pressure gradient (Figure 6b). As a result, the flow started to separate towards the mid-section of the tag, leading to a large wake behind the tag. High pressure differentials are also observed along the surface of the tag, generating large lift.

The results of Model A showed a significant improvement from Model 0, achieving a drag reduction of $39 \%$ and lift reduction of $48 \%$ compared to Model 0. Due to its pointed shape and smooth body, it could maintain attached streamlines throughout the whole contour of the tag (Figure 4a),
Fig. 3:

- Graph of drag force of the final design (Model D), a control (Model 0) and three other iterations at differing speeds.

- All models were tested at three speeds $(1,3,5 \mathrm{~m} / \mathrm{s})$ on CFD
Fig. 2:

- Graph of lift force of the final design (Model D), a control (Model 0 ) and three other iterations at differing speeds.

- All models were tested at three speeds $(1,3,5 \mathrm{~m} / \mathrm{s})$ on $\mathrm{CFD}$
When the extruded canards were added in Model B, the lift was further reduced by $8 \%$ compared to Model A. However, with the indented canards, a $68 \%$ lift reduction could be achieved compared to Model B. Both the canards generated high pressure at the top of the tag and negative pressure at the bottom (Figure 5). Additionally, they created vortices that deflected the flow downwards, reducing lift further. (Figure 4e). However, it was observed that the canards caused flow separation after the midsection of the tag (Figure 4b).

The dimples in Model C generated low-pressure vortices across the tag, reducing the size of the wake observed in Model. Interestingly, the dimples also accounted for a reduction in lift by $22 \%$. In Model D, higher base (back of the tag) pressure and spanwise vortices (Figure 4e) were produced because of the tabs. In addition, the 
streamlined hydrophones generated more uniform pressure gradients. Therefore, both the tabs and hydrophones helped achieve pressure drag reduction. Overall, Model D achieved a reduction in drag by $56 \%$ (Figure 3 ) and lift by 86\% (Figure 2) compared to Model 0.

\section{Discussion}

While biologging devices have revolutionized ecology, ocean management, and climate change monitoring, they can be an impediment towards the animal's movement. This can lead to an increased chance of nest abandonment, lowered swimming speeds while foraging, lowered oxygen consumption as well as faulty data collection [8]. The present work explores new design elements to add to the DTAG3 housing, to reduce the hydrodynamic loading that marine animals experience. This final design was able to reduce drag by $56 \%$ and lift by $86 \%$ when compared to Model 0 at $5 \mathrm{~m} / \mathrm{s}$. The final design was reached through iterative tests done with 3D CAD models and a simplified simulated flow environment using a CFD analysis.

Tests on the existing GPS Phone Tag CAD model showed (Figure 6a) (Model 0) that large drag and lift was generated (Figure 6bc) due to its abrupt form changes, sharp

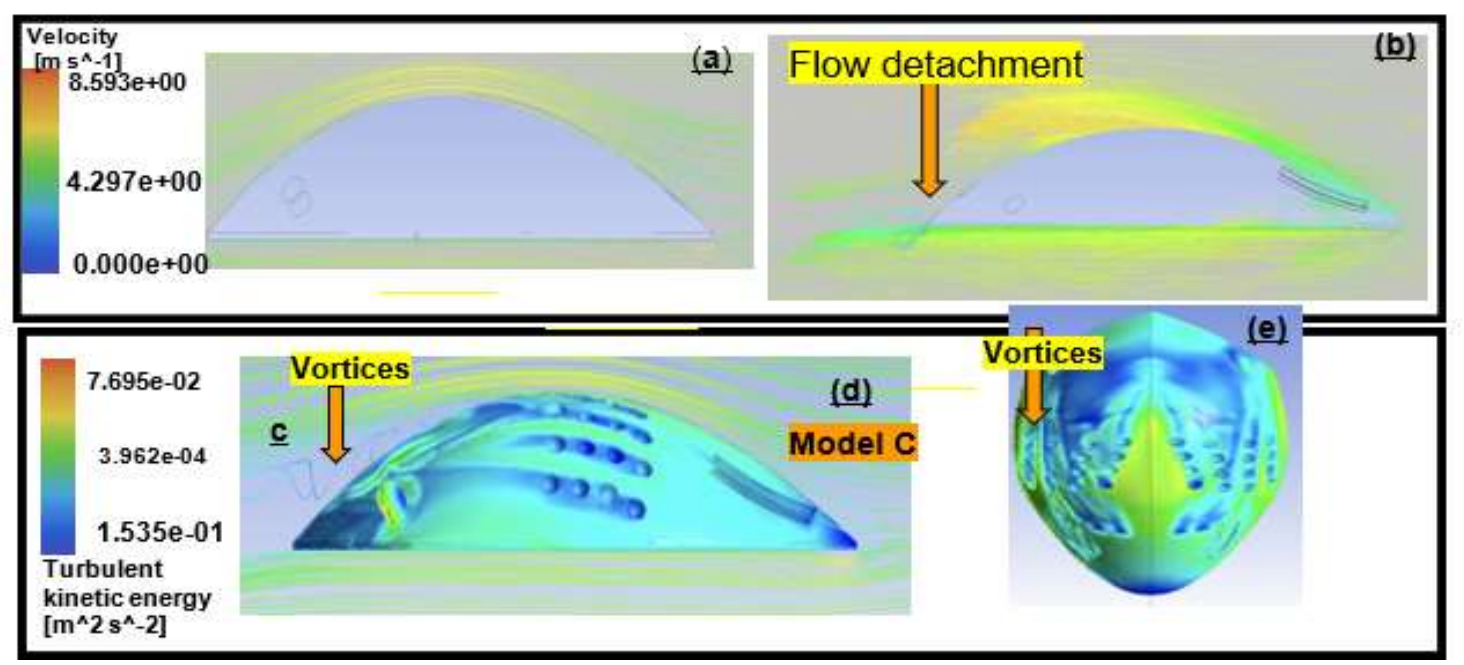

fig. 4: CFD simulation results of all four iterations at $5 \mathrm{~m} / \mathrm{s}$ flow

(a, b) Longitudinal cross section view of streamlines and vectors along tag with a color map, where areas of red are high velocity and areas of blue are low velocity

(c, d) Model C streamlines with surface turbulent kinetic energy contours

(e) Top view of turbulent kinetic energy contours around Model D where light blue areas indicate areas of vortex generation corners, and a large frontal profile, leading to flow separation and high-pressure differentials. These results were similar to those of [10], who tested a similar design. Therefore, Model A could achieve significant drag (39\%) and lift (48\%) reduction when compared to Model 0, owing to its smooth contour and narrow front.

The canards introduced in Model B and Model D could further reduce lift by up to $77 \%$ from Model A through generating negative pressure at the bottom of the tag, and vortices that could deflect some flow downwards. They were made as indented shapes to avoid possible entanglements with vegetation that could occur while deployed on an animal. However, the canards were responsible for considerable flow separation. A reason for this is because the canards serve as disturbances to the flow over the otherwise smooth tag housing, so a disproportionate amount of flow through the canards is being directed upwards on the tag. These effects were akin to those observed with the channel and spoiler elements included in the design presented by [3].

Flow separation introduced in Model B was delayed with the inclusion of dimples in Model $\mathrm{C}$, playing a similar role as they do in golf balls. They were, however, responsible for marginal drag reduction. A possible reason for this is that they were added on top of a hydrodynamic housing, whereas dimples are traditionally included to reduce drag on blunt bodies [16]. However, the dimples accounted for significant lift reduction. This could be due to vortices 
generated on the edges of the dimples intensifying the lift reducing effect of the canards, through collectively increasing the vortical strength. Such a synergy was also discussed in [3], where their inverted wing
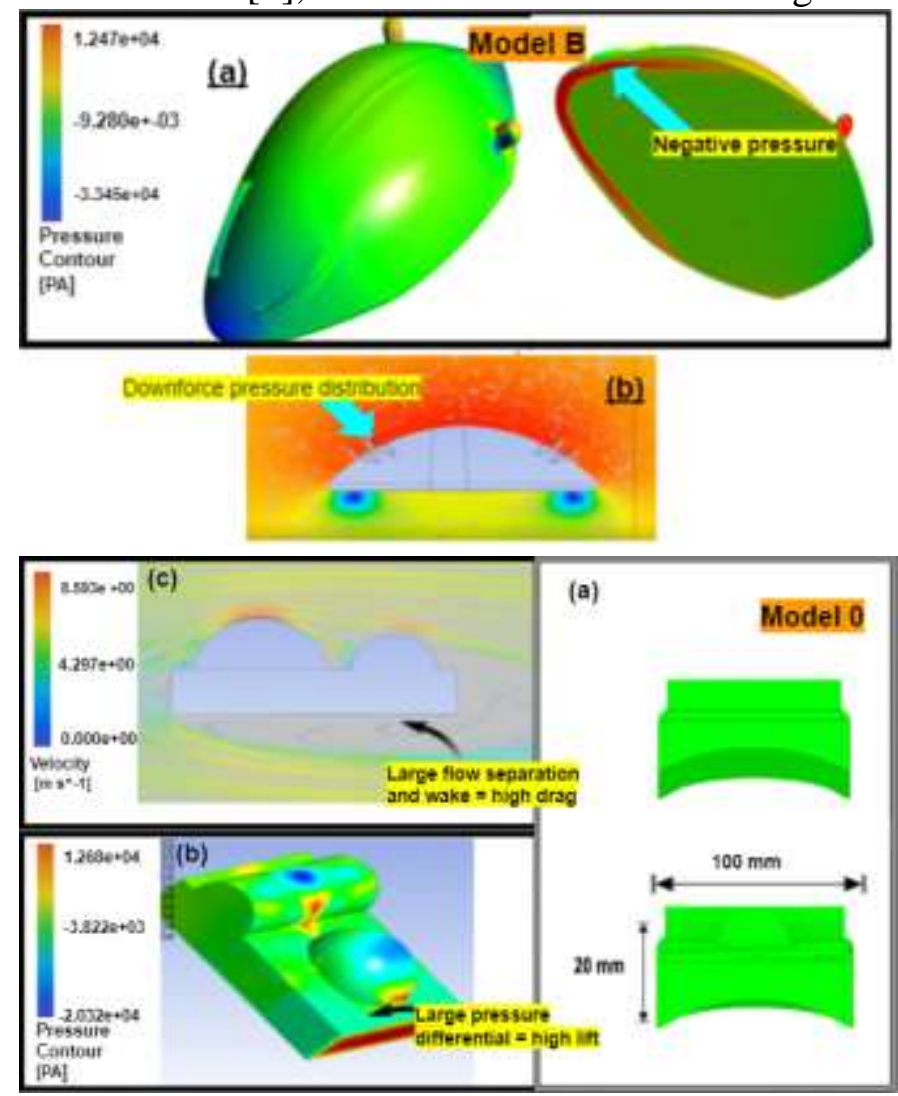

base pressure. Additionally, spanwise vortices generated from the tabs may have also been responsible for the further lift reduction when compared to Model C. The new forms of hydrophones also accounted for more uniform pressure gradients, though the practical implementation of the streamlined hydrophones depends on the specific electronic constraints that different types of tags have.

CFD modelling can be improved in the future by using a sinusoidally varying flow inlet (front of domain where flow enters) to model the relative flow around the animal. Additionally, the most accurate turbulence models, chosen based on experimental results, can be used for the top and bottom halves of the tag [18].

\section{CONCLUSION}

The objective of this study was to design a new biologging tag form that houses the DTAG3 electronics and reduces the total lift and drag, to element enhanced the performance of their channel component.

The tabs in Model D successfully reduced the pressure drag through generating higher

Fig. 5:

- (a) GPS Phone tag CAD (Model 0) model that was used as control in experiments

- Pressure contour and streamline diagrams outputted from the CFD simulations indicate that the tag induced (c) pressure differentials and (b) large flow separation respectively

Fig. 6: CFD simulation results of Model B at $5 \mathrm{~m} / \mathrm{s}$ flow with pressure color contours

- surface contours with negative pressure regions at bottom of tag

- horizontal cross section at front of tag showing the low-pressure differential generated because of the canards avoid the risk of faulty data being relayed, and the tagged animal's biomechanics being impeded. A new design (Model D) has been developed that was able to meet all the design criteria set. The tag was able to reduce drag by $56 \%$ and lift by $86 \%$ compared to Model 0 . This was through the use of an elliptical nose shape and a slowly converging tail shape to allow flow recovery; indented canards to reduce frontal lift; tabs to reduce pressure drag; and more streamlined hydrophone shapes. Finally, dimples were included to further reduce the drag. There was an observed synergy between all the design elements, contributing to a further lift reduction.

This tag design can be further enhanced and tested through more advanced testing with CFD, and wind-tunnel testing with $3 \mathrm{D}$ printed tags at different orientations and sizes. Designs should be modified to account for specific animals and their physical changes throughout stages of their lives. Tag induced drag and lift should be tested on 
different animals by running in-situ tests of the Model D, in a variety of scenarios, to study longterm impacts.

\section{REFERENCES}

[1] S. J. Bograd, B. A. Block, D. P. Costa, and B. J. Godley, "Biologging technologies: new tools for conservation. Introduction,” Endangered Species Research, vol. 10, pp. 1-7, 2010.

[2] N. E. Hussey, S. T. Kessel, K. Aarestrup, S. J. Cooke, P. D. Cowley, A. T. Fisk, R. G. Harcourt, K. N. Holland, S. J. Iverson, J. F. Kocik, J. E. Mills Flemming, and F. G. Whoriskey, "Aquatic animal telemetry: A panoramic window into the underwater world," Science, vol. 348, no. 6240, pp. $1255642-$ $1255642,2015$.

[3] G. Fiore, E. Anderson, C. S. Garborg, M. Murray, M. Johnson, M. J. Moore, L. Howle, and K. A. Shorter, "From the track to the ocean: Using flow control to improve marine bio-logging tags for cetaceans," PLOS ONE, vol. 12, no. 2, 2017.

[4] D. A. Rosen, C. G. Gerlinsky, and A. W. Trites, "Telemetry tags increase the costs of swimming in northern fur seals,Callorhinus ursinus," Marine Mammal Science, vol. 34, no. 2, pp. 385-402, 2017.

[5] R. P. Wilson, N. R. Coria, H. J. Spairani, D. Adelung, and B. Culik, "Human-induced behaviour in Adelie penguins Pygoscelis adeliae," Polar Biology, vol. 10, no. $1,1989$.

[6] R. Wilson and C. McMahon, "Measuring devices on wild animals: what constitutes acceptable practice?," Frontiers in Ecology and the Environment, vol. 4, no. 3, pp. 147-154, 2006.

[7] D. A. Croll, S. D. Osmek, and J. L. Bengtson, "An Effect of Instrument Attachment on Foraging Trip Duration in Chinstrap Penguins," The Condor, vol. 93, no. 3, pp. 777-779, 1991 .

[8] D. Zhang, J. M. Hoop, V. Petrov, J. Rocho-Levine, M. J. Moore, and K. A. Shorter, "Simulated and experimental estimates of hydrodynamic drag from biologging tags," Marine Mammal Science, vol. 36, no. 1, pp. 136-157, 2019.

[9] N. Jespen, "A brief discussion of the $2 \%$ tag/bodymass rule," Aquatic Telemetry: Advances and Applications. Proceedings of the Fifth Conference on Fish Telemetry Held in Europe. , 2004.

[10] W. P. Kay, D. S. Naumann, H. J. Bowen, S. J. Withers, B. J. Evans, R. P. Wilson, T. B. Stringell, J. C. Bull, P. W. Hopkins, and L. Börger, "Minimizing the impact of biologging devices: Using computational fluid dynamics for optimizing tag design and positioning,"
Methods in Ecology and Evolution, vol. 10, no. 8, pp. 1222-1233, 2019.

[11] D. B. Morton, P. Hawkins, R. Bevan, K. Heath, J. Kirkwood, P. Pearce, L. Scott, G. Whelan, and A. Webb, "Refinements in telemetry procedures: Seventh report of BVAAWF/FRAME/RSPCA/UFAW Joint Working Group on Refinement, Part A," Laboratory Animals, vol. 37, no. 4, pp. 261-299, 2003.

[12] J. Katz , Race Car Aerodynamics: Designing for Speed (Engineering and Performance), 2nd ed. .

[13] K. Alex Shorter, M. M. Murray, M. Johnson, M. Moore, and L. E. Howle, "Drag of suction cup tags on swimming animals: Modeling and measurement," Marine Mammal Science, vol. 30, no. 2, pp. 726-746, 2013.

[14] M. Moonesun, M. Asghar, K. Mikhailovich, D. Mehdi, and J. Moshref, "Concepts in submarine shape design.," Concepts in submarine shape design, vol. 45, no. 1, pp. 100-104, 2016.

[15] M. Gallego, "Design, simulation and testing of a downforce-increasing car bodykit," Air Navigation Engineering, 2019.

[16] K. Aoki, K. Muto, and H. Okanga , “AERODYNAMIC CHARACTERISTIC AND FLOW PATTERN ON DIMPLES STRUCTURE OF A SPHERE. ," Flucome, vol. 10, no. 1, pp. 2-10, 2009.

[17] H. Park, D. Lee, W. Jeon, S. Hahn, J. Kim, J. Kim, J. Choi, H. Choi, Drag reduction in flow over a twodimensional bluff body with a blunt trailing edge using a new passive device," Journal of Fluid Mechanics, vol 563, p. 389, 2006.

[18] A. Kyte, C. Pass, R. Pemberton, M. Sharman, and J. C. McKnight, "A computational fluid dynamics (CFD) based method for assessing the hydrodynamic impact of animal borne data loggers on host marine mammals," Marine Mammal Science, vol. 35, no. 2, pp. 364-394, 2018. 